\title{
Association of Low Heart Rate Variability with Atherosclerotic Cardiovascular Disease in Hemodialysis Patients
}

\author{
J.C. Longenecker ${ }^{a, b} \quad$ M. Zubaid ${ }^{b, c} \quad$ K.V. Johny ${ }^{b, c} \quad$ A.I. Attiac J. Alic W. Rashed ${ }^{c}$ \\ C.G. Suresh ${ }^{c}$ M. Omar ${ }^{b, c}$ \\ aDepartment of Community Medicine, Faculty of Medicine, Health Sciences Centre, Kuwait University, \\ ${ }^{\mathrm{b}}$ Department of Medicine, Faculty of Medicine, Kuwait University, and 'Department of Medicine, \\ Mubarak Al-Kabeer Hospital, Kuwait
}

\section{Key Words}

Heart rate variability - End-stage renal disease $\cdot$ Dialysis $\cdot$

Atherosclerotic cardiovascular disease $\cdot$ Echocardiography

\begin{abstract}
Objective: The aim of this study was to investigate the leftventricular (LV) mass-adjusted association between low heart rate variability (HRV) and atherosclerotic cardiovascular disease (ASCVD) among hemodialysis patients in Kuwait. Subjects and Methods: One hundred and eight patients were enrolled in the study. HRV time domain measures were obtained by 48-hour Holter monitoring, including the standard deviation of all R-wave-to-R-wave (RR) intervals (SDNN), standard deviation of all 5-min averaged intervals (SDANN), HRV triangular index (HRV-TI), percent of adjacent RR intervals differing by $>50 \mathrm{~ms}$ ( $\mathrm{pNN50)}$ ), and root mean square of sums of squares of all differences (rMSSD). Left ventricular ejection fraction (LVEF) and LV mass index (LVMI) were measured by M-mode echocardiography. Comorbidity was assessed using medical record review. Prevalent ASCVD was defined as coronary artery, cerebrovascular, or peripheral vascular disease. Results: Prevalence of ASCVD, LV hypertrophy, and LVEF $<40 \%$ were 56,59 , and $10 \%$, respectively. The SDANN was negatively associated with ASCVD (-20 ms; $\mathrm{p}=$ $0.003)$, LV systolic dysfunction ( $-20 \mathrm{~ms} ; \mathrm{p}=0.001)$, elevated
\end{abstract}

LVMI (-20 ms; $p=0.002)$, hypertension ( $-34 \mathrm{~ms} ; \mathrm{p}=0.01)$, and diabetes $(-20 \mathrm{~ms} ; \mathrm{p}=0.001)$. After adjustment for hypertension and LVMI using logistic regression, ASCVD was associated with the lowest quartile of SDANN (OR $=4.3, p=0.009)$, HRV-TI (OR = 3.3, $p=0.03)$, and SDNN (OR = 2.3, $p=0.10)$. These associations persisted after adjusting for LVEF. Conclusion: In dialysis patients, low HRV indices were strongly associated with prevalent ASCVD, independent of LVMI and LVEF. The interrelationships among HRV, diabetes, hypertension, and LVMI should be addressed in studies of HRV and ASCVD.

Copyright $\odot 2009$ S. Karger AG, Basel

\section{Introduction}

The risk of atherosclerotic cardiovascular disease (ASCVD) and cardiovascular mortality is over 10 times higher in dialysis patients than in the general population [1], but the increased prevalence of traditional ASCVD risk factors in dialysis patients only explains part of this increased risk [2]. Therefore, many research studies in dialysis patients have focused on nontraditional risk factors for ASCVD [1], including reduced heart rate variability (HRV).

\section{KARGER}

Fax +4161306 1234

E-Mail karger@karger.ch

www.karger.com
(C) 2009 S. Karger AG, Basel

1011-7571/09/0182-0085\$26.00/0

Accessible online at:

www.karger.com/mpp
J. Craig Longenecker, $\mathrm{MD}, \mathrm{PhD}, \mathrm{MPH}$

Department of Community Medicine, Faculty of Medicine

Health Sciences Centre, Kuwait University

PO Box 24923, 13110 Safat (Kuwait)

Tel. +965 531 2000, ext. 6534, Fax +965 533 8948, E-Mail jlongene@hsc.edu.kw 
Small oscillations in the RR interval duration (time between two R waves on an ECG) occur normally, resulting in beat-to-beat variability in the heart rate. Autonomic nervous system activity directly affects these oscillations [3]. Various standard HRV measures, which measure different components of the autonomic nervous system, decrease in the setting of increased sympathetic tone, reduced parasympathetic tone, or autonomic dysfunction [3]. In the general population, large studies have reported associations between decreased HRV and an increased risk of coronary heart disease (CHD), independent of traditional risk factors [4-6].

HRV is decreased in end-stage renal disease patients compared to healthy controls [7, 8], and HRV in dialysis patients increases significantly after renal transplantation [9]. These findings suggest that the decreased HRV in end-stage renal disease may be an important nontraditional risk factor and event predictor for ASCVD. Supporting this conclusion, several small prospective studies of dialysis patients have shown that low HRV predicts allcause mortality [10], sudden death [10], or cardiac-related death $[11,12]$. These studies, however, were limited by the lack of information regarding the confounding effects of left ventricular hypertrophy (LVH) in the relationship between HRV and ASCVD [13]. It remains unknown whether the reduced HRV is simply a marker for LVH or an ASCVD risk factor independent of left ventricular (LV) indices.

This study extends the current knowledge regarding HRV and ASCVD by including measurements of LV structure and function, allowing assessment of the association between HRV and ASCVD independent of potential confounding effects of LV mass. The a priori hypothesis is that low HRV will be associated with prevalent ASCVD, even after adjusting for the confounding effects of LV mass index (LVMI) and LV ejection fraction (LVEF).

\section{Subjects and Methods}

\section{Study Participants}

From June, 2005, to May, 2007, 115 chronic hemodialysis patients in the outpatient Dialysis Unit at Mubarak Al-Kabeer Hospital in Al-Jabriya, Kuwait, were approached for this cross-sectional study, of whom 108 were enrolled (94\% participation rate). The only exclusion criteria were pacemaker placement, atrial fibrillation, and inability to give informed consent. The study protocol adhered to the Declaration of Helsinki and was approved by the Ethics Committees of the Faculty of Medicine and the Kuwait Institute for Medical Specialization. All participants provided written, informed consent prior to data collection.

\section{Data Collection}

Sociodemographic characteristics and health behaviors were obtained by staff-administered questionnaires. Blood pressure was taken using a standard mercury manometer immediately prior to the enrollment dialysis session. Clinical data were obtained from physician review of medical records. Mention of a condition (past or present) in the medical record was sufficient for positive coding. Prevalent ASCVD was defined as a history of CHD (myocardial infarction, coronary bypass/angioplasty, or angina), cerebrovascular disease (stroke, carotid endarterectomy, or recurrent transient ischemic attack), or peripheral vascular disease (peripheral artery angioplasty or bypass, inoperable peripheral vascular disease, or aortic aneurysm). Congestive heart failure was defined as a history of pulmonary edema or a diagnosis of congestive heart failure in the medical record. Diabetes included both type I and type II diabetes.

Holter monitoring was performed using the Delmar Reynolds LifeCard $\mathrm{CF}^{\circledR}$ Digital Holter Monitor (three ECG leads). The Holter monitor was placed $30 \mathrm{~min}$ after the enrollment dialysis session, and removed $48 \mathrm{~h}$ later, after the next session. Echocardiography using two-dimensional M-Mode (General Electric Vivid 7 using an M3S probe) was used to determine LV internal diameters in diastole (LVIDd) and systole, septal/posterior wall thicknesses, LV dysfunction, wall motion abnormalities, and LVEF. Septal wall thickness of $11 \mathrm{~mm}$ and posterior wall thickness of $9 \mathrm{~mm}$ were used to define the upper limit of normal wall thickness. LV mass was calculated using the Penn Convention [14] where: $\operatorname{LV}$ mass $(\mathrm{g})=1.04\left[(\mathrm{LVIDd}+\mathrm{sw}+\mathrm{pw})^{3}-(\text { LVIDd })^{3}\right]$ - $13.6 \mathrm{~g}$. LVMI $\left(\mathrm{g} / \mathrm{m}^{2}\right)$ was calculated as the LV mass standardized to body surface area. Criteria for LVH included LVMI $>135 \mathrm{~g} / \mathrm{m}^{2}$ for men, and LVMI $>111 \mathrm{~g} / \mathrm{m}^{2}$ for women. A low LVEF was defined as an ejection fraction below $40 \%$.

\section{Analysis of $H R V$}

Holter tracings were scanned using the Delmar Reynolds Impressario ${ }^{\circledR}$ software (version 2.6.17). We chose the time domain measures to assess HRV, according to the recommendation of the Task Force of the European Society of Cardiology for long tracings ( $\geq 24 \mathrm{~h}$ ) [3]. Ectopic beats such as premature ventricular contractions or ventricular tachycardia were excluded from analysis. Time domain measures which correspond to total HRV (reflecting a combination of lower sympathetic and higher parasympathetic/vagal tone) included: the standard deviation (SD) of all normal RR intervals (SDNN), the SD of the mean normal RR intervals in all 5-min segments of the tracing (SDANN), and HRV triangular index (HRV-TI; the total number of all RR intervals divided by the height of the histogram of RR intervals measured on a discrete scale with bins of $1 / 128 \mathrm{~s}$ ). Time domain measures which primarily reflect parasympathetic/vagal tone included: the square root of the mean of the sums of squares of the differences of all adjacent RR intervals (rMSSD) and the percentage of successive normal RR interval pairs differing by more than $50 \mathrm{~ms}$ (pNN50). The process of analyzing HRV tracings was blinded to all other study data.

\section{Statistical Analysis}

All analyses were performed using Stata version 8.0. Twotailed $\mathrm{p}$ values for bivariate analyses were generated by $\mathrm{t}$ test, ANOVA, Mann-Whitney U test, or Pearson $\chi^{2}$, where appropriate. To assess associations of other variables with the HRV, it was 
Fig. 1. Scatterplots and correlation matrix between HRV indices. Spearman correlations $\left(r_{s}\right)$ and $p$ values are provided for each correlation pair.

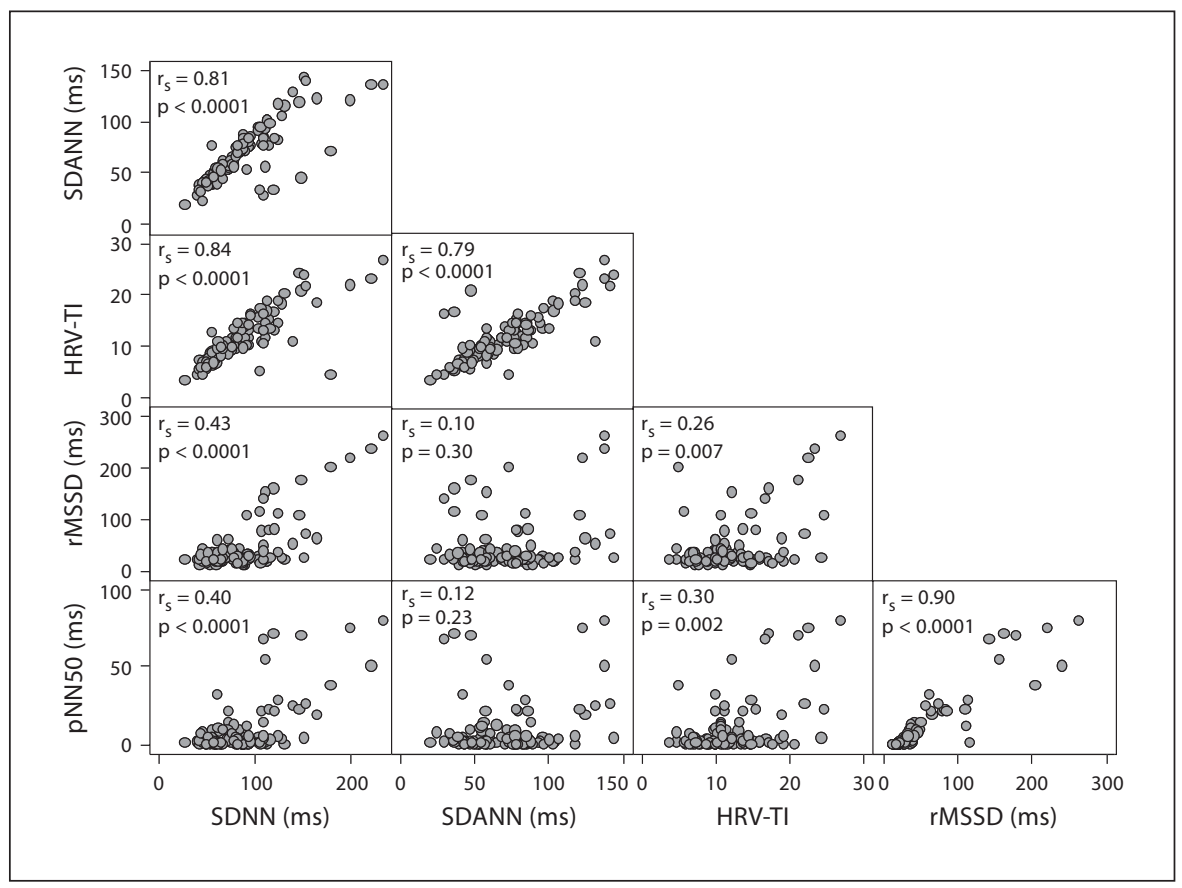

decided a priori to analyze each HRV index as (a) a continuous variable, (b) a dichotomous variable divided at the median value, and (c) a categorical variable divided into quartiles. Associations between the highly skewed continuous HRV indices and other variables were assessed using multiple median regression (the qreg command in Stata [15]), with each HRV measure as the dependent variable (there is no assumption of normality for median regression). The coefficients generated by these models represent the absolute difference in median values of the dependent variable in the comparison groups. For analyses of ASCVD, logistic regression (with prevalent ASCVD as the binary dependent variable) was used to obtain adjusted odds ratios. The lowest quartile $\left(Q_{1}\right)$ of each HRV measure was compared to the second to fourth quartiles. Owing to the relatively small number of ASCVD events, a maximum of three independent variables were entered simultaneously into regression models. LVEF and LVMI were entered into the models as quartile variables, to avoid assumptions of linearity. A priori analyses stratified by diabetes were performed and statistical interaction determined by entering a diabetes interaction term into the model.

\section{Results}

Median duration since the start of dialysis among the 108 patients was 1.9 years (range, $0.0-20.1$ years), with $97 \%$ of participants having a duration less than 10 years. All participants had a 48-hour Holter monitor trace, echocardiographic measurements, and a medical record review. The median percentage of qualified beats for all
ECG tracings was 94.5\%. The median time between Holter placement and echocardiogram was 14 days. Medical record review revealed no major cardiovascular events between the Holter and echocardiogram procedures. The prevalence levels of ASCVD, LVH and low LVEF were 56, 59 , and $10 \%$, respectively. The prevalence of $\mathrm{CHD}$, cerebrovascular disease, and peripheral vascular disease were 51,16 , and $17 \%$, respectively.

Nonparametric (Spearman) correlations between the five time-domain measures of HRV are shown in figure 1. As expected, very strong significant Spearman correlations ranging from 0.79 to 0.84 were seen among SDNN, SDANN, and HRV-TI (which reflect both higher vagal and lower sympathetic activity) and a correlation of 0.90 between rMSSD and pNN50 (which primarily reflect vagal activity).

Participants' clinical and echocardiographic characteristics and their associations with prevalent ASCVD are presented in table 1 . Upon enrollment, $90.7 \%$ had a history of hypertension, $61.1 \%$ were diabetic and $22.2 \%$ had a history of congestive heart failure. Prevalent ASCVD was significantly associated with older age, congestive heart failure, and diabetes. Median SDNN, SDANN, and HRV-TI were all significantly lower among patients with a history of ASCVD, compared to those without ASCVD. 
Table 1. Participant characteristics and associations with prevalent ASCVD

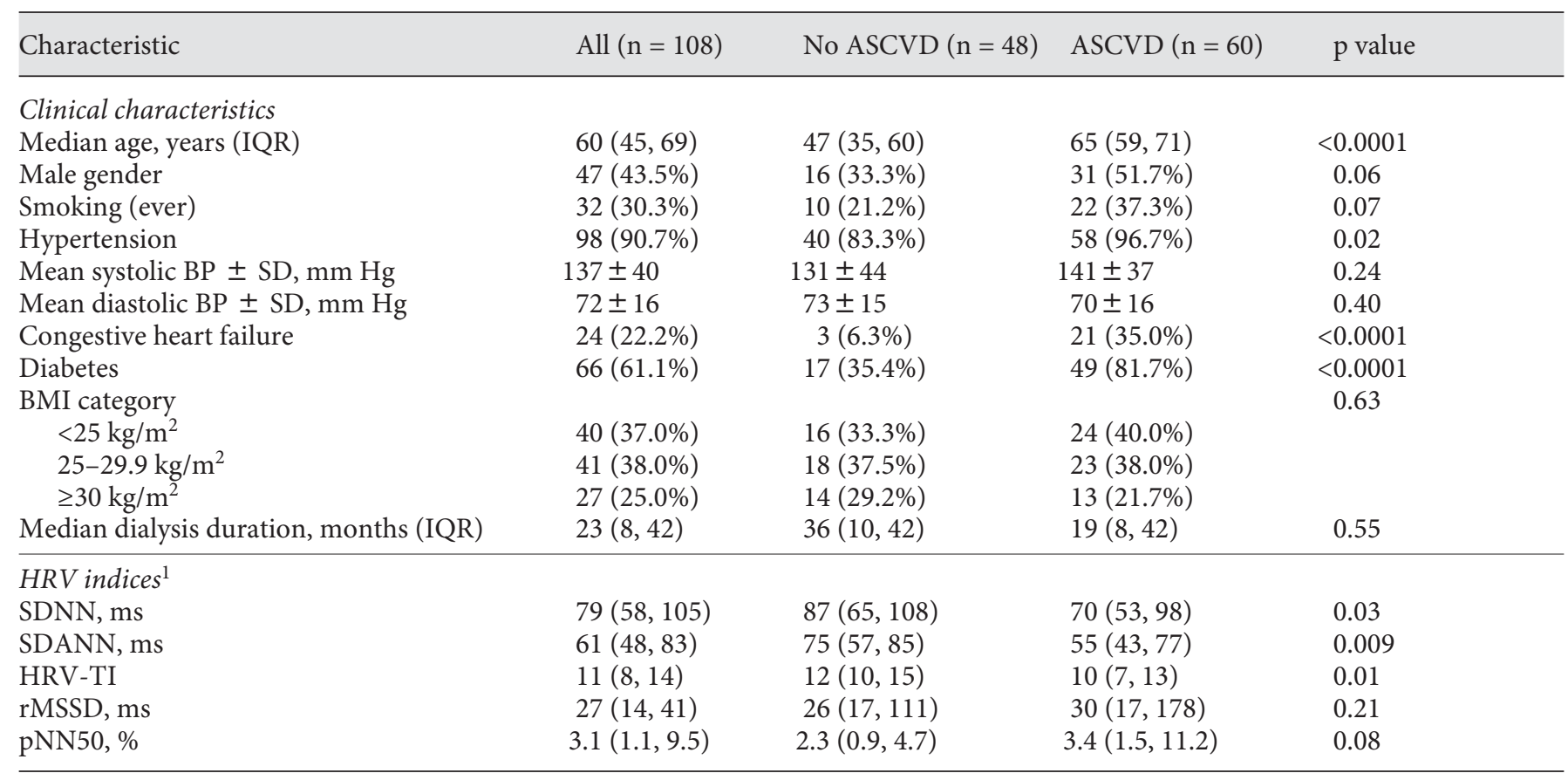

$\mathrm{p}$ values generated by $\mathrm{t}$ test, Mann-Whitney $\mathrm{U}$ test, or Pearson $\chi^{2}$, where appropriate, for differences by ASCVD status. IQR $=$ Interquartile range (values at the 25 th and 75 th percentile); $\mathrm{BP}=$ blood pressure; $\mathrm{BMI}=$ body mass index.

${ }^{1}$ Median and IQR (in parentheses).

The associations of HRV indices with clinical and echocardiographic characteristics are presented in table 2. Diabetes, hypertension, and ASCVD were significantly associated with lower median SDNN and SDANN, and marginally lower HRV-TI. Of the echocardiographic indices, only LVH, defined as elevated LVMI, was significantly associated with a lower median SDNN, SDANN, and HRV-TI. However, LV dysfunction was significantly associated with a lower SDANN, and a thickened posterior wall with a lower HRV-TI. Older age was associated with higher rMSSD $(+12 \mathrm{~ms} ; \mathrm{p}=0.003)$ and pNN50 (+4.0; $\mathrm{p}=0.04)$. Neither rMSSD nor pNN50, however, were associated with any other characteristics.

Strong associations between ASCVD and the lowest quartile $\left(\mathrm{Q}_{1}\right)$ of SDNN, SDANN, and HRV-TI (compared to quartiles 2-4; $\mathrm{Q}_{2-4}$ ) were found, with unadjusted odds ratios in the range of 2.9-5.0 (table 3). Significant associations persisted after adjustment for LVEF, LVMI, and hypertension. Although moderate associations remained after adjustment for diabetes, they became statistically not significant. There were no significant associations between ASCVD and either rMSSD or pNN50. No interaction by diabetes was present. Adjustment for duration on dialysis demonstrated minimal confounding effects. Because of the nonspecificity of the diagnosis of angina pectoris in dialysis patients, we performed a separate analysis which excluded 'angina-only' from the definition of ASCVD. This analysis resulted only in minor differences in the odds ratio estimates.

The unadjusted and adjusted associations between prevalent ASCVD and SDNN, SDANN, and HRV-TI quartiles, respectively, are presented in figure 2 . The strongest associations between these HRV measures and ASCVD occurred in the lowest quartile, with some evidence of mild confounding by LVMI, and further confounding by hypertension.

\section{Discussion}

This study assessed the cross-sectional associations between time-domain HRV indices and prevalent ASCVD, with particular attention paid to the potential confounding effects of LV mass and LVEF. We found strong associations between ASCVD and SDNN, SDANN, and HRV-TI, which remained independent of LVMI and 
Table 2. Associations of HRV indices with clinical characteristics and echocardiographic indices

\begin{tabular}{llll}
\hline \multirow{2}{*}{ Participant characteristics } & \multicolumn{3}{l}{ Difference in median } \\
\cline { 2 - 4 } & $\begin{array}{l}\text { SDNN } \\
\text { ms }\end{array}$ & $\begin{array}{l}\text { SDANN } \\
\text { ms }\end{array}$ & HRV-TI \\
& & & \\
\hline Clinical characteristics & +2 & -12 & -0.5 \\
Age $(>58$ vs. $\leq 58$ years $)$ & +4 & +5 & -0.5 \\
Gender (F vs. M) & $-18^{\ddagger}$ & -11 & -1.1 \\
Current smoking & $-14^{\ddagger}$ & $-20^{* *}$ & $-2.2^{\ddagger}$ \\
Diabetes & $-32^{*}$ & $-34^{*}$ & $-4.4^{*}$ \\
History of hypertension & $-22^{* *}$ & $-20^{* * *}$ & -1.9 \\
Currently hypertensive & $-16^{*}$ & $-20^{* *}$ & $-1.8^{\ddagger}$ \\
ASCVD & -14 & -9 & -1.3 \\
Congestive heart failure & & & \\
Echocardiographic indices & -24 & -16 & -3.2 \\
Ejection fraction $<40 \%$ & -8 & -12 & -0.5 \\
Septal wall $>11$ mm & -12 & -15 & $-3.7^{* *}$ \\
Posterior wall $>9$ mm & $-19^{*}$ & $-20^{* *}$ & $-3.6^{* *}$ \\
LV hypertrophy & $-17^{*}$ & $-20^{* *}$ & -1.3 \\
LV dysfunction & $-23^{\ddagger}$ & $-17^{*}$ & -1.3 \\
Wall motion abnormality & & & \\
\hline
\end{tabular}

Statistical significance: ${ }^{\ddagger} \mathrm{p}<0.10 ;{ }^{*} \mathrm{p}<0.05 ;{ }^{*} \mathrm{p}<0.01$; ${ }^{* * *} \mathrm{p}<0.001$; coefficients and $\mathrm{p}$ values generated by median regression models.

${ }^{1} \mathrm{LV}$ hypertrophy defined for men as LVMI $>135 \mathrm{~g} / \mathrm{m}^{2}$, and for women as LVMI $>111 \mathrm{~g} / \mathrm{m}^{2}$. No significant differences in rMSSD or pNN50 were seen for the clinical history variables or echocardiographic indices (data not shown).

LVEF after adjustment for confounding. The LV mass, LVEF, and hypertension produced, at most, moderate confounding effects in the associations between ASCVD and HRV indices, whereas diabetes had stronger confounding effects. These data extend the understanding of $\mathrm{HRV}$ in the dialysis population by evaluating the role of LV size and function in those associations.

\section{HRV and Cardiovascular Disease}

The relationship between HRV and ASCVD is complex. Studies have shown that HRV is decreased in the acute phase of myocardial infarction, but appears to return to normal afterwards [16]. Immediately after myocardial infarction, a low HRV also is a strong predictor of mortality independent of its association with cardiac failure [17-20]. Assessing low HRV as a risk factor for CHD in the general population, both the Framingham Heart Study [4] and the ARIC Study [5] reported low HRV indices as risk factors for CHD independent of traditional risk factors. The Framingham Heart Study found that a
Table 3. Association between HRV indices and prevalent ASCVD among 108 dialysis patients

\begin{tabular}{|c|c|c|}
\hline \multirow[t]{2}{*}{ Logistic regression models } & \multicolumn{2}{|c|}{ Odds ratio of ASCVD } \\
\hline & estimate & $95 \% \mathrm{CI}$ \\
\hline \multicolumn{3}{|l|}{ SDNN models $\left(Q_{1} \text { vs. } Q_{2-4}\right)^{1}$} \\
\hline Unadjusted & $2.9^{*}$ & $1.1,7.7$ \\
\hline \multicolumn{3}{|l|}{ Adjusted for } \\
\hline LVEF & $2.6^{\ddagger}$ & $1.0,7.2$ \\
\hline LVMI & $2.5^{\ddagger}$ & $0.9,6.9$ \\
\hline LVMI and diabetes & 1.7 & $0.6,5.1$ \\
\hline LVMI and hypertension & 2.3 & $0.8,6.5$ \\
\hline \multicolumn{3}{|l|}{ SDANN models $\left(Q_{1} \text { vs. } Q_{2-4}\right)^{1}$} \\
\hline Unadjusted & $5.0^{* *}$ & $1.7,14.4$ \\
\hline \multicolumn{3}{|l|}{ Adjusted for } \\
\hline LVEF & $4.3^{*}$ & $1.4,12.9$ \\
\hline LVMI & $4.7^{* *}$ & $1.5,14.0$ \\
\hline LVMI and diabetes & $3.0^{\ddagger}$ & $0.9,9.6$ \\
\hline LVMI and hypertension & $4.3^{* *}$ & $1.4,13.0$ \\
\hline \multicolumn{3}{|l|}{ HRV-TI models $\left(Q_{1} \text { vs. } Q_{2-4}\right)^{1}$} \\
\hline Unadjusted & $3.2^{*}$ & $1.2,8.2$ \\
\hline \multicolumn{3}{|l|}{ Adjusted for } \\
\hline LVEF & $3.0^{*}$ & $1.0,8.6$ \\
\hline LVMI & $3.1^{*}$ & $1.1,8.8$ \\
\hline LVMI and diabetes & 1.7 & $0.5,5.4$ \\
\hline LVMI and hypertension & $3.3^{*}$ & $1.1,9.8$ \\
\hline
\end{tabular}

Statistical significance: ${ }^{*} \mathrm{p}<0.10 ;{ }^{*} \mathrm{p}<0.05 ;{ }^{* *} \mathrm{p}<0.01$.

${ }^{1}$ Comparison groups are $\mathrm{Q}_{1}$ versus $\mathrm{Q}_{2-4}$ for SDANN, SDNN, and HRV-TI, respectively. No interaction by diabetes was present.

1-SD decrease in SDNN (but not rMSSD or pNN50) was associated with new $\mathrm{CHD}$ events $(\mathrm{RH}=1.45 ; 95 \% \mathrm{CI} 1.13$, 1.85). The ARIC Study also found that the increased risk of CHD associated with low HRV measures was seen primarily in the lowest quartile of the HRV measures, consistent with our findings that the association is strongest in the lowest quartile of HRV measures (fig. 2). These studies, among others, have engendered interest in the potential role of HRV in the increased ASCVD risk associated with end-stage renal disease.

\section{$H R V$ and ASCVD in End-Stage Renal Disease}

Since uremia is associated with a significantly decreased HRV [7-9], attention has been given to the potential role that decreased HRV may have in the increased ASCVD risk in dialysis patients. Results from three small prospective cohort studies, which followed dialysis patients from 2 to 5 years demonstrated that SDNN, 

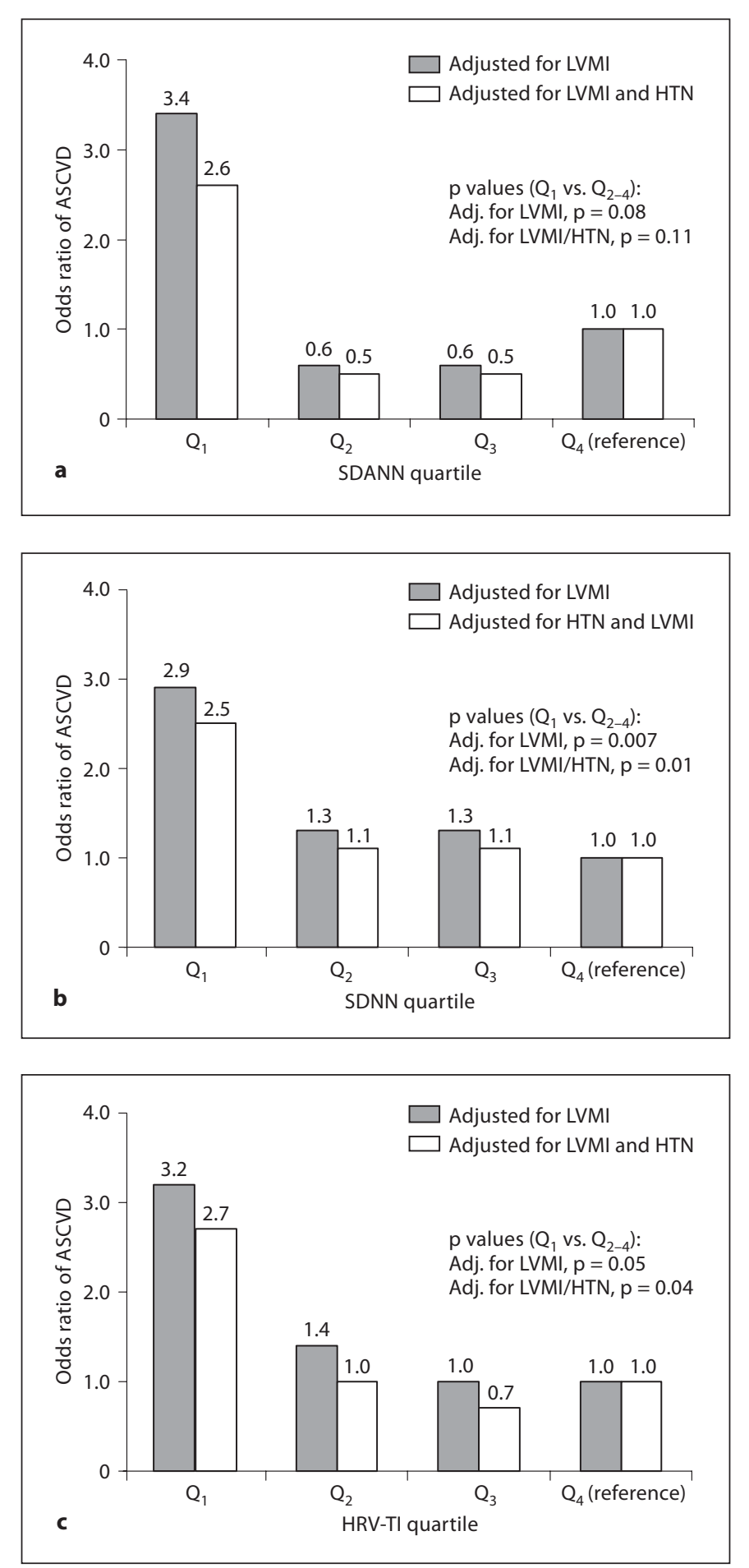

Fig. 2. Unadjusted and adjusted associations of HRV indices with prevalent ASCVD. HTN = Hypertension. a SDANN. b SDNN. c HRV-TI.
HRV-TI, SDANN, and/or ultra-low frequency HRV power (which correlates with SDANN) strongly predict cardiac-related mortality, reporting significant relative risks in the 1.9 to 12.6 range $[10,11,21]$. Conversely, these studies showed that rMSSD, pNN50, and/or high-frequency HRV power (which is correlated with rMSSD and pNN50) do not predict cardiac-related death. Consistent with these studies, we found that SDNN, SDANN, and HRVTI, but not pNN50 or rMSSD, are associated with ASCVD.

It should be noted that only one [10] of the three prospective studies of HRV in dialysis patients adjusted for the potential confounding effects of LV ejection fraction, and none of them adjusted their analyses for LV mass. Because LVMI is associated with HRV [13] (the exposure variable of interest) and with ASCVD (the outcome variable of interest), it has potential to confound the association between HRV and ASCVD. For this reason, we adjusted the association between HRV and ASCVD for LVMI and LVEF. Our findings demonstrate that LV mass and ejection fraction in fact do produce small to moderate positive confounding effects in the cross-sectional association between low HRV and prevalent ASCVD. The association between HRV and ASCVD persisted after adjustment for these LV structure and function parameters.

\section{Clinical Implications}

As an independent risk factor and predictor of ASCVD, low HRV has the potential to be used to identify dialysis patients at higher levels of risk for ASCVD. These patients could be targeted for more intensive cardiovascular risk reduction, more frequent surveillance for ASCVD, earlier referral for cardiac catheterization, or other more intensive preventive efforts. Even if low HRV were simply a marker for ASCVD among patients with end-stage renal disease, it may be useful as a tool for risk stratification, particularly if the very high relative risks reported by small prospective studies are confirmed in larger studies.

On the other hand, if low HRV is causally associated with ASCVD and/or sudden death, then improvement of HRV itself would have potential to reduce cardiovascular risk. Indeed, studies have shown that beta-blockers, calcium channel blockers, angiotensin-converting enzyme inhibitors, and angiotensin receptor blockers can increase HRV indices in humans [22]. Also, a cohort study among dialysis patients surviving cardiac arrest found all these classes of medications to be associated with increased survival [23]. However, no randomized trials in 
dialysis patients have yet been performed. It is therefore currently unknown whether or not improving HRV itself would improve survival in dialysis patients, or whether the potential benefit of treatment with these medications would be mediated through lowering HRV or through other mechanisms. Future randomized trials should test the hypothesis that improvement of HRV among dialysis patients with low HRV indices will decrease risk of ASCVD and sudden death.

\section{Potential Mechanisms}

Our findings suggest that sympathetic overstimulation and parasympathetic withdrawal may together be important in the outcomes of ASCVD and death. Whether this effect is primarily one of decreasing cardiac electrochemical stability (thereby increasing the risk of ventricular fibrillation and sudden death), or accelerating atherosclerotic processes, or both, remains unknown. Hayano et al. [10] found a much higher relative risk of sudden death than a relative risk of cardiac death, providing indirect evidence that one mechanism through which low HRV is mediated is fatal arrhythmias. However, both the Framingham Study [4] and the ARIC Study [5] were performed among individuals without heart disease at baseline, suggesting that sympathetic overstimulation and/or parasympathetic withdrawal may be involved in the mechanisms of atherosclerosis itself, a hypothesis supported by our findings. Future studies should investigate potential mechanisms which may link decreased HRV and atherosclerosis.

\section{Limitations}

In any cross-sectional study, inferences regarding associations should be evaluated cautiously, owing to the possibility of reverse causality. However, the interpretation that low HRV is a risk factor for ASCVD is logically made in this study because it is consistent with the findings of previously published prospective studies. It is also recognized that the sample size of this study is not large, resulting in some wide confidence intervals. Large studies of HRV and ASCVD in the dialysis population will be needed in the future, particularly cohort studies and randomized controlled trials of medications that alter HRV indices. The study population was derived from the largest outpatient dialysis clinic in Kuwait, with fair generalizability to other dialysis patients in Kuwait.

\section{Conclusion}

This cross-sectional study of 108 hemodialysis patients found strong associations between low HRV measures and prevalent ASCVD, after adjusting for the small to moderate confounding effects of LV mass and LV ejection fraction. We conclude that low HRV indices in hemodialysis patients, particularly SDNN, SDANN, and HRV-TI, which reflect a combination of sympathetic overstimulation and parasympathetic withdrawal, are associated with prevalent atherosclerotic ASCVD, independent of LV size and function.

\section{Acknowledgments}

We are grateful for the consistent and diligent efforts of Ms. Mona Zeidan and Dr. Mary Said, who were involved with patient enrollment and data collection, and Dr. Ali Boushahri, who also collected clinical data. Supporting grant: No. MC01/03, Research Administration, Kuwait University.

\section{References}

1 Foley RN, Parfrey PS, Sarnac MJ: Clinical epidemiology of cardiovascular disease in chronic renal disease. Am J Kidney Dis 1998; 32:S112-S119.

-2 Sarnak MJ, Levey AS, Schoolwerth AC, Coresh J, Culleton B, Hamm LL, McCullough PA, Kasiske BL, Kelepouris E, Klag MJ, Parfrey P, Pfeffer M, Raij L, Spinosa DJ, Wilson PW: Kidney disease as a risk factor for development of cardiovascular disease: a statement from the American Heart Association Councils on Kidney in Cardiovascular Disease, High Blood Pressure Research, Clinical Cardiology, and Epidemiology and Prevention. Circulation 2003;108:2154-2169.
-3 Heart rate variability: standards of measurement, physiological interpretation and clinical use. Task Force of the European Society of Cardiology and the North American Society of Pacing and Electrophysiology. Circulation 1996;93:1043-1065.

-4 Tsuji H, Larson MG, Venditti FJ Jr, Manders ES, Evans JC, Feldman CL, Levy D: Impact of reduced heart rate variability on risk for cardiac events. The Framingham Heart Study. Circulation 1996;94:2850-2855.
5 Liao D, Cai J, Rosamond WD, Barnes RW, Hutchinson RG, Whitsel EA, Rautaharju P, Heiss G: Cardiac autonomic function and incident coronary heart disease: a populationbased case-cohort study. The ARIC Study. Atherosclerosis Risk in Communities Study. Am J Epidemiol 1997; 145:696-706.

-6 Tsuji H, Venditti FJ Jr, Manders ES, Evans JC, Larson MG, Feldman CL, Levy D: Determinants of heart rate variability. J Am Coll Cardiol 1996;28:1539-1546.

7 Steinberg AA, Mars RL, Goldman DS, Percy RF: Effect of end-stage renal disease on decreased heart rate variability. Am J Cardiol 1998;82:1156-1158, A10. 
$>8$ Kurata C, Uehara A, Sugi T, Ishikawa A, Fujita K, Yonemura K, Hishida A, Ishikawa K, Tawarahara K, Shouda S, Mikami T: Cardiac autonomic neuropathy in patients with chronic renal failure on hemodialysis. Nephron 2000;84:312-319.

$>9$ Yildiz A, Sever MS, Demirel S, Akkaya V, Turk S, Turkmen A, Ecder T, Ark E: Improvement of uremic autonomic dysfunction after renal transplantation: a heart rate variability study. Nephron 1998;80:57-60.

10 Hayano J, Takahashi H, Toriyama T, Mukai S, Okada A, Sakata S, Yamada A, Ohte N, Kawahara $\mathrm{H}$ : Prognostic value of heart rate variability during long-term follow-up in chronic haemodialysis patients with endstage renal disease. Nephrol Dial Transplant 1999;14:1480-1488.

11 Fukuta H, Hayano J, Ishihara S, Sakata S, Mukai S, Ohte N, Ojika K, Yagi K, Matsumoto H, Sohmiya S, Kimura G: Prognostic value of heart rate variability in patients with end-stage renal disease on chronic haemodialysis. Nephrol Dial Transplant 2003;18: 318-325.

$\checkmark 12$ Hathaway DK, Cashion AK, Milstead EJ, Winsett RP, Cowan PA, Wicks MN, Gaber AO: Autonomic dysregulation in patients awaiting kidney transplantation. Am J Kidney Dis 1998;32:221-229.
13 Nishimura M, Hashimoto T, Kobayashi H, Fukuda T, Okino K, Yamamoto N, Nakamura $\mathrm{N}$, Yoshikawa $\mathrm{T}$, Takahashi $\mathrm{H}$, Ono $\mathrm{T}$ : Association between cardiovascular autonomic neuropathy and left ventricular hypertrophy in diabetic haemodialysis patients. Nephrol Dial Transplant 2004;19: 2532-2538.

14 Muiesan ML, Salvetti M, Monteduro C, Donato F, Rizzoni D, Agabiti-Rosei E: Various ways of calculating echocardiographic left ventricular mass and their relative prognostic values. J Hypertens 1998;16:1201-1206.

15 StataCorp: qreg - Quantile (including median) Regression; in Stata Reference Manual, release 7.0. College Station, Stata Press, 2001, vol 3, pp 11-27.

-16 Casolo GC, Stroder P, Signorini C, Calzolari F, Zucchini M, Balli E, Sulla A, Lazzerini S: Heart rate variability during the acute phase of myocardial infarction. Circulation 1992; 85:2073-2079.

17 Fei L, Copie X, Malik M, Camm AJ: Shortand long-term assessment of heart rate variability for risk stratification after acute myocardial infarction. Am J Cardiol 1996;77: 681-684.
8 Bigger JT Jr, Fleiss JL, Steinman RC, Rolnitzky LM, Kleiger RE, Rottman JN: Frequency domain measures of heart period variability and mortality after myocardial infarction. Circulation 1992;85:164-171.

19 Kop WJ, Verdino RJ, Gottdiener JS, O’Leary ST, Bairey Merz CN, Krantz DS: Changes in heart rate and heart rate variability before ambulatory ischemic events (1). J Am Coll Cardiol 2001;38:742-749.

20 Casolo GC, Stroder P, Sulla A, Chelucci A, Freni A, Zerauschek M: Heart rate variability and functional severity of congestive heart failure secondary to coronary artery disease. Eur Heart J 1995;16:360-367.

21 Cashion AK, Holmes SL, Arheart KL, Acchiardo SR, Hathaway DK: Heart rate variability and mortality in patients with end stage renal disease. Nephrol Nurs J 2005;32:173184

22 Ranpuria R, Hall M, Chan CT, Unruh M: Heart rate variability (HRV) in kidney failure: measurement and consequences of reduced HRV. Nephrol Dial Transplant 2008; 23:444-449.

23 Pun PH, Lehrich RW, Smith SR, Middleton JP: Predictors of survival after cardiac arrest in outpatient hemodialysis clinics. Clin J Am Soc Nephrol 2007;2:491-500. 